\title{
Three eras of micellization
}

\author{
J. C. $\mathrm{Neu}^{*}$ \\ Department of Mathematics, University of California at Berkeley, Berkeley, California 94720 \\ J. A. Cañizo ${ }^{\dagger}$ \\ Departamento de Matemática Aplicada, Universidad de Granada, E-18071 Granada, Spain \\ L. L. Bonilla \\ Departamento de Matemáticas, Universidad Carlos III de Madrid, Avenida Universidad 30, E-28911 Leganés, Spain
}

(Received 24 August 2002; published 30 December 2002)

\begin{abstract}
Micellization is the precipitation of lipids from aqueous solution into aggregates with a broad distribution of aggregation number. Three eras of micellization are characterized in a simple kinetic model of Becker-Döring type. The model asigns the same constant energy to the $(k-1)$ monomer-monomer bonds in a linear chain of $k$ particles. The number of monomers decreases sharply and many clusters of small size are produced during the first era. During the second era, nuclei are increasing steadily in size until their distribution becomes a self-similar solution of the diffusion equation. Lastly, when the average size of the nuclei becomes comparable to its equilibrium value, a simple mean-field Fokker-Planck equation describes the final era until the equilibrium distribution is reached.
\end{abstract}

DOI: 10.1103/PhysRevE.66.061406

PACS number(s): 82.70.Uv, 83.80.Qr, 05.40.-a, 05.20.Dd

\section{INTRODUCTION}

Spontaneous self-assembly of small molecular aggregates in aqueous solutions forms association colloids or complex fluids [1]. Depending on their mean aggregation number, molecular volume, and critical hydrocarbon chain length, lipids can pack into spherical or cylindrical micelles. The surfaces of these structures are formed by the hydrophilic heads of the monomer molecules, whose hydrophobic tails lie inside the aggregate. Equilibrium thermodynamics shows that rodlike cylindrical aggregates have a polydisperse distribution of sizes (micellization), whereas the sizes of spherical aggregates grow indefinitely (phase segregation) [1]. The latter process is similar to other examples of first order phase transitions [2] such as condensation of liquid droplets from a supersaturated vapor, colloidal crystallization [3], and the segregation by coarsening of binary alloys quenched into the miscibility gap [4-6]. Understanding the kinetics of nucleation and growth beyond the determination of the steadystate nucleation rate is a task of great importance and not yet completely accomplished. This is so despite a rich literature on nucleation and growth [7], and several attempts at bridging the gap between nucleation and late-stage coarsening theories [8].

In this paper, we study asymptotically a simple discrete model of micellization kinetics of Becker-Döring type [7-10]. Starting from an initial condition of pure monomers, we expect the system to evolve to the well-known polydisperse equilibrium distribution [1]. However, the nonequilibrium evolution is interesting per se and because the method-

\footnotetext{
*Email address: neu@math.berkeley.edu

†Email address: ozarfreo@yahoo.com

*Author to whom correspondence should be addressed. Email address: bonilla@ing.uc3m.es
}

ology employed here may be applicable to the kinetics of phase segregation. We find that the approach to equilibrium occurs in three well-defined stages or eras. Starting from the initial state of pure monomers, the number of monomers decreases sharply and many clusters of small size are produced during the first era. During the second era, aggregates are increasing steadily in size until their distribution becomes a self-similar solution of the diffusion equation. Lastly, when the average size of the nuclei becomes comparable to its equilibrium value, a simple mean-field Fokker-Planck equation describes the final era until the equilibrium distribution is reached. Numerical solution of the model confirms all the theoretical predictions.

The rest of the paper is as follows. In Sec. II, we review the equilibrium properties of self-assembling aggregates and introduce discrete kinetic models of Becker-Döring type to describe them. Depending on the binding energy of the aggregate with $k$ monomers ( $k$ cluster), micellization or phase segregation occurs. For rodlike aggregates, the binding energy of a $k$ cluster (relative to isolated monomers in solution) is $(k-1)$ times the monomer-monomer bond energy, and an equilibrium size distribution exists (micellization). For spherical aggregates, the binding energy includes a term proportional to the surface area of the aggregate and no equilibrium size distribution exists beyond a critical density. Then aggregates grow indefinitely and phase segregation occurs following the typical nucleation and growth kinetics. Section III presents a numerical simulation of micellization kinetics, which clearly reveals its three eras. The agenda of the asymptotic analysis is now clear, and is carried out in Sec. IV. The last Sec. V contains our conclusions and suggestions for experiments.

\section{THERMODYNAMICS AND KINETIC MODELS}

The model presented here is nucleation in a lattice. There are systems, such as proteins aggregating in a cubic phase of 
lipid bilayers, for which a lattice formulation is physically correct. In this paper, the main reasons for a lattice model are clarity, and the expectation that the dilute limit of the lattice model (in which there are many more binding sites $M$ than particles $N$ ) should closely resemble crystallization from a dilute solution. The latter is a classical problem in the kinetic theory of first-order phase transitions [2]. We shall now review the equilibrium statistical mechanics of aggregates, distinguishing between micellization and phase segregation, and then introduce the kinetic models we study.

\section{A. Equilibrium size distribution of aggregates}

Let us assume that we have $p_{k} \geqslant 0$ clusters with $k$ particles (in short, $k$ clusters), so that

$$
N=\sum_{k=1}^{N} k p_{k} .
$$

Let $e_{k}$ be the energy of a $k$ cluster. The total energy of the lattice system is

$$
E=\sum_{k=1}^{N} p_{k} e_{k}=N e_{1}+\sum_{k=2}^{N} p_{k}\left(e_{k}-k e_{1}\right)
$$

where we have used the particle conservation (2.1). Except for a constant $N e_{1}$, the total energy is

$$
\begin{gathered}
E=-\sum_{k=2}^{N} p_{k} \varepsilon_{k}, \\
\varepsilon_{k}=k e_{1}-e_{k} .
\end{gathered}
$$

Now $E$ is the total lattice energy measured with respect to a configuration in which all clusters are monomers, and $\varepsilon_{k}$ is the binding energy of the $k$ cluster (notice the sign convention). We will obtain the equilibrium configuration by minimizing the free energy density with respect to the density of $k$ clusters. To calculate the entropy, we proceed as follows. Let $n_{j} \geqslant 0$ be the occupation number of the site $j, j$ $=1, \ldots, M$. The configuration space of the lattice consists of all $M$-tuples of occupation numbers $\left\{n_{1}, \ldots, n_{M}\right\}$, with $\sum_{j=1}^{M} n_{j}=N$ and $N \ll M$. Clearly, there are many indistinguishable configurations that produce the same given set of numbers $p_{1}, \ldots, p_{N}$. Their number $\Omega$ is given by the BoseEinstein counting argument,

$$
\Omega=\frac{M !}{p_{1} ! \cdots p_{N} !\left(M-p_{1}-\cdots-p_{N}\right) !},
$$

and the entropy of the system is $k_{B} \ln \Omega$. In the appropriate thermodynamic limit, $N \rightarrow \infty$ with fixed densities $\rho \equiv N / M$ (particles) and $\rho_{k} \equiv p_{k} / M$ ( $k$ clusters), particle conservation becomes

$$
\sum_{k=1}^{\infty} k \rho_{k}=\rho,
$$

and we can show that the entropy density is

$$
\begin{gathered}
\mathcal{S} \equiv \frac{k_{B}}{M} \ln \Omega \sim-k_{B}\left(\sum_{k=1}^{\infty} \rho_{k} \ln \rho_{k}+r \ln r\right), \\
r=1-\sum_{k=1}^{\infty} \rho_{k},
\end{gathered}
$$

by using Stirling's formula. The free energy density $f$ $=E / M-T \mathcal{S}$ can be written in terms of $\rho$ and the densities of clusters having two or more particles by using its definition and Eqs. (2.3) and (2.6)-(2.8). The result is

$$
f=-\sum_{k=2}^{\infty} \rho_{k} \varepsilon_{k}+k_{B} T \sum_{k=1}^{\infty} \rho_{k} \ln \rho_{k}+k_{B} \operatorname{Tr} \ln r,
$$

where $\rho_{1}=\rho-\sum_{k=2}^{\infty} k \rho_{k}$ and $r=1-\sum_{k=1}^{\infty} \rho_{k}$. In the dilute limit, $\quad 1-r=\sum_{k=1}^{\infty} \rho_{k}<\sum_{k=1}^{\infty} k \rho_{k}=\rho \ll 1$, and therefore $r$ $\sim 1, r \ln r \sim-\sum_{k=1}^{\infty} \rho_{k}$, and Eq. (2.9) becomes

$$
f=-\sum_{k=2}^{\infty} \rho_{k} \varepsilon_{k}+k_{B} T \sum_{k=1}^{\infty} \rho_{k}\left(\ln \rho_{k}-1\right),
$$

which corresponds to the Boltzmann counting. The equilibrium density of $k$ clusters $(k \geqslant 2)$ can be found by differentiating this equation with respect to $\rho_{k}$ and equating the result to zero. Taking into consideration that $\partial \rho_{1} / \partial \rho_{k}=-k$ $(k \geqslant 2)$, we obtain

$$
\tilde{\rho}_{k}=\rho_{1}^{k} \exp \left(\frac{\varepsilon_{k}}{k_{B} T}\right),
$$

the positive sign in the argument of the exponential is due to our definition of the binding energies. Equation (2.11) can be rewritten as

$$
\begin{gathered}
\tilde{\rho}_{k}=\exp \left(-\frac{g_{k}}{k_{B} T}\right), \\
g_{k}=-\varepsilon_{k}+k_{B} T k \ln \left(\frac{1}{\rho_{1}}\right) ;
\end{gathered}
$$

$g_{k}$ as a function of $k$ can be interpreted as the activation energy of nucleation theory. The equilibrium density of monomers can be found by inserting Eq. (2.11) into Eq. (2.6) and solving the resulting self-consistent equation for $\rho_{1}$ in terms of the constant density $\rho$ :

$$
\sum_{k=1}^{\infty} k \rho_{1}^{k} \exp \left(\frac{\varepsilon_{k}}{k_{B} T}\right)=\rho .
$$

Whether this self-consistent equation has a solution depends on the value of $\rho$ and on the model we adopt for the binding energy of a $k$ cluster. Typical models are as follows. For rodlike aggregates,

$$
\varepsilon_{k}=(k-1) \alpha k_{B} T,
$$

where $\alpha k_{B} T$ is the monomer-monomer bonding energy [1]. For spherical aggregates, 


$$
\varepsilon_{k} \sim(k-1) \alpha k_{B} T-\frac{3}{2} \sigma k^{2 / 3}
$$

for $k \gg 1$. Here $\sigma=2 \gamma\left(4 \pi v^{2} / 3\right)^{1 / 3}$, where $\gamma$ and $v=V / M$ are the interfacial free energy per unit area (surface tension) and the molecular volume, respectively.

Inserting Eq. (2.15) in Eq. (2.14) and using $\sum_{k=1}^{\infty} k x^{k}$ $=x(d / d x) \sum_{k=1}^{\infty} x^{k}=x /(1-x)^{2}$, we obtain

$$
\rho=\frac{\rho_{1}}{\left(1-\rho_{1} e^{\alpha}\right)^{2}} .
$$

This equation has the unique solution

$$
\rho_{1}=\frac{1+2 \rho e^{\alpha}-\sqrt{1+4 \rho e^{\alpha}}}{2 \rho e^{2 \alpha}},
$$

with $\rho_{1}<e^{-\alpha}$ for all values of the density $\rho[1]$. Notice that

$$
\langle k\rangle \equiv \frac{\sum_{k=1}^{\infty} k \tilde{\rho}_{k}}{\sum_{k=1}^{\infty} \tilde{\rho}_{k}}=\frac{\sqrt{1+4 \rho e^{\alpha}}-1}{2}
$$

is the average cluster size in equilibrium. Notice that for $\rho e^{\alpha} \gg 1,\langle k\rangle \sim \sqrt{\rho e^{\alpha}}$ and $\tilde{\rho}_{k} \sim e^{-\alpha} e^{-k /\langle k\rangle}$.

For spherical aggregates, the self-consistency condition based on the approximation to $\varepsilon_{k}$ in Eq. (2.16) is

$$
\rho_{1} \sum_{k=1}^{\infty} k\left(\rho_{1} e^{\alpha}\right)^{k-1} \exp \left(-\frac{3 \sigma k^{2 / 3}}{2 k_{B} T}\right)=\rho .
$$

Clearly, this series converges, provided $\rho_{1} e^{\alpha}<1$; and it diverges if $\rho_{1} e^{\alpha}>1$. The critical monomer concentration $\rho_{1}$ $=e^{-\alpha}$ is called critical micelle concentration (CMC) [1]. Below CMC, Eq. (2.20) can be solved for $\rho_{1}$, and the aggregates eventually form micelles with an equilibrium size distribution, whereas phase segregation and indefinite aggregate growth results if more monomers are added above the CMC. For $k \gg 1$, the free energy (2.13) is $g_{k} \sim \alpha k_{B} T+3 \sigma k^{2 / 3} / 2$ $-k \varphi$, with $\varphi=k_{B} T \ln \left(\rho_{1} e^{\alpha}\right)$. For $\varphi>0, g_{k}$ increases for small $k$, it has a maximum at the critical cluster size $k_{c}$ $\approx(\sigma / \varphi)^{3}$, and then it decays monotonically as $k$ further increases.

\section{B. Kinetic models}

Let us now formulate the kinetic theory of aggregation in these systems. As in the Becker-Döring kinetic theory, we shall assume that a $k$ cluster can grow or decay by capturing or shedding one monomer at a time. Then

$$
\begin{aligned}
& \dot{\rho}_{k}=j_{k-1}-j_{k} \equiv-D_{-} j_{k}, \quad k \geqslant 2, \\
& j_{k}=d_{k}\left\{e^{\left(D_{+} \varepsilon_{k}\right) / k_{B} T} \rho_{1} \rho_{k}-\rho_{k+1}\right\},
\end{aligned}
$$

or finally,

$$
j_{k}=d_{k}\left\{\left(e^{-\left(D_{+} g_{k}\right) / k_{B} T}-1\right) \rho_{k}-D_{+} \rho_{k}\right\},
$$

Here $D_{+} \varepsilon_{k} \equiv \varepsilon_{k+1}-\varepsilon_{k}=-D_{+} g_{k}+k_{B} T \ln \left(1 / \rho_{1}\right)$ and $j_{k}$ is the net rate of creation of a $k+1$ cluster from a $k$ cluster, given by the mass action law. We have made the detailed balance assumption to relate the kinetic coefficient for monomer aggregation to that of decay of a $(k+1)$ cluster, $d_{k}$. Then $\tilde{\rho}_{k}$ given by Eq. (2.11) solves $j_{k}=0$. The kinetic model is described by a closed system of equations once we supplement Eqs. (2.6), (2.21), and (2.22) with expressions for the binding energy of a $k$ cluster, $\varepsilon_{k}$, and for the kinetic coefficient of the decay reaction, $d_{k}$.

The simplest possible model for micellization within the Becker-Döring theory is obtained by setting $\varepsilon_{k}=(k$ $-1) \alpha k_{B} T$ and $d_{k}=1$ in Eq. (2.22) for the creation rate of a $(k+1)$ cluster (rescaling of time can absorb a constant cluster decay rate $d_{k}=d$; typical time scales describing aggregation kinetics range from microseconds to milliseconds [1113]). Equations (2.21) and (2.22) then become the following discrete Smoluchowski equation:

$$
\dot{\rho}_{k}+\left(e^{\alpha} \rho_{1}-1\right)\left(\rho_{k}-\rho_{k-1}\right)=\rho_{k+1}-2 \rho_{k}+\rho_{k-1},
$$

to be solved together with conservation condition (2.6), namely, $\sum_{k=1}^{\infty} k \rho_{k}=\rho$. At $t=0$, we assume that $\rho_{k}=\rho \delta_{k 1}$. We shall consider the limit $\rho \gg e^{-\alpha}$, in which the initial monomer concentration is much larger than the CMC. The parameters $\rho$ and $\alpha$ are not really independent. If we rescale the cluster densities with $\rho$, so that

$$
\rho_{k}=\rho r_{k},
$$

and define a scaled time

$$
\tau \equiv e^{\alpha} \rho t \equiv \frac{t}{\epsilon}
$$

the rescaled problem contains the single parameter $\epsilon$ $\equiv\left(\rho e^{\alpha}\right)^{-1} \ll 1$. Then Eqs. (2.24) and (2.6) become

$$
\begin{gathered}
\frac{d r_{k}}{d \tau}+\left(r_{1}-\epsilon\right)\left(r_{k}-r_{k-1}\right)=\epsilon\left(r_{k+1}-2 r_{k}+r_{k-1}\right), \quad k \geqslant 2 \\
1=\sum_{k=1}^{\infty} k r_{k},
\end{gathered}
$$

to be solved with initial conditions

$$
r_{1}(0)=1, \quad r_{2}(0)=r_{3}(0)=\cdots=0 .
$$

Lastly, notice that we can straightforwardly derive two global identities from Eqs. (2.27) and (2.28):

$$
\begin{gathered}
\frac{d r_{1}}{d \tau}+r_{1}\left(r_{1}+r_{c}\right)+\epsilon\left(r_{1}-r_{2}-r_{c}\right)=0, \\
\frac{d r_{c}}{d \tau}+r_{1} r_{c}+\epsilon\left(r_{1}-r_{c}\right)=0 .
\end{gathered}
$$

Here $r_{c}$ is the total density of clusters, 

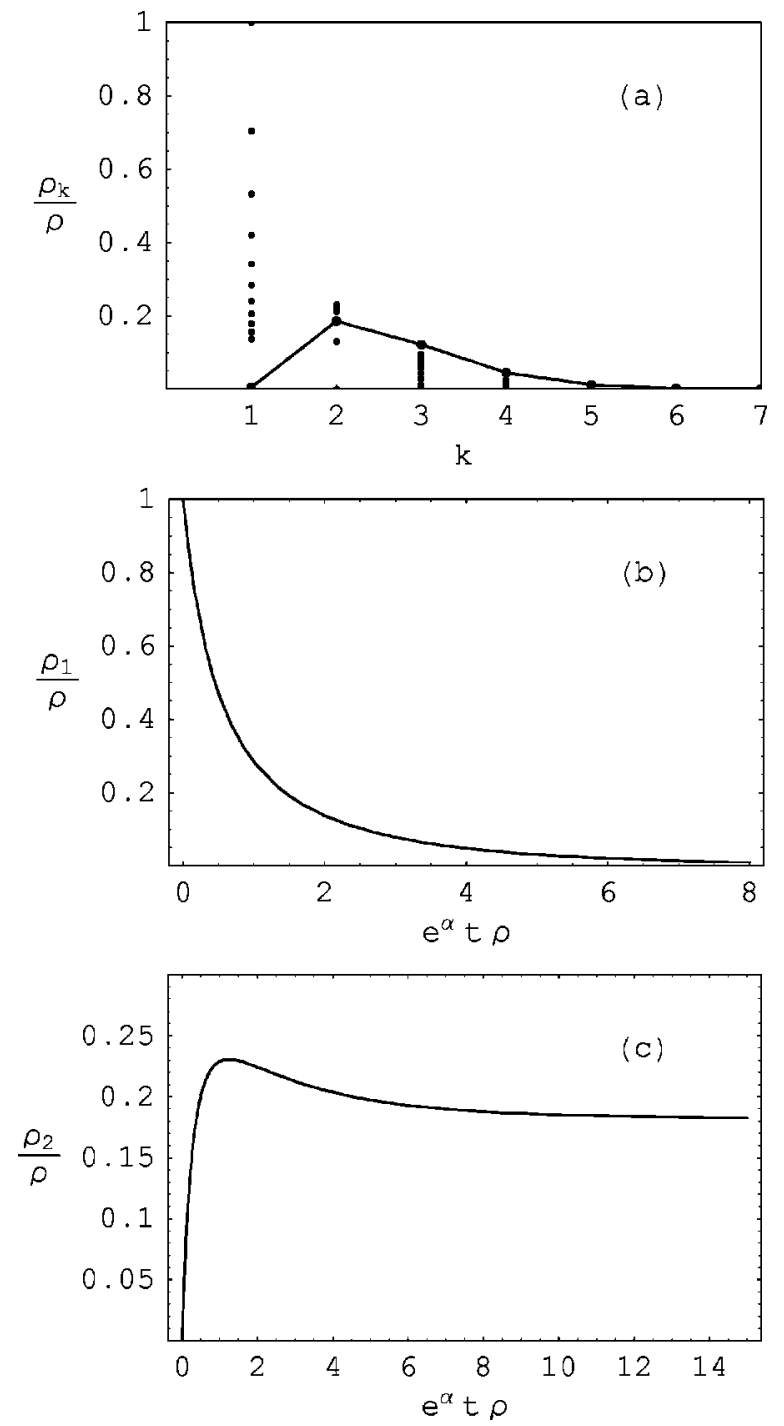

FIG. 1. (a) Scaled cluster size distribution $\rho_{k} / \rho$ as a function of $k$ for $0 \leqslant \tau \leqslant 10$. At time $\tau=10$, the values of $\rho_{1} / \rho, \rho_{2} / \rho$, etc. have been joined by straight lines as a guide to the eye. (b) Evolution of the scaled monomer concentration $\rho_{1} / \rho$. (c) Evolution of the scaled dimer concentration $\rho_{2} / \rho$. Parameter values are $\alpha=10$ and $\rho$ $=0.1$.

$$
r_{c}=\sum_{k=1}^{\infty} r_{k},
$$

and initially, $r_{c}(0)=1$.

\section{NUMERICAL RESULTS}

Numerical solution of the initial value problem given by Eqs. (2.27)-(2.29) clearly expresses the phenomenology of micellization, and informs the singular perturbation analysis carried out in Sec. IV. Figures 1-5 illustrate the evolution of the size distribution for $\epsilon=4.54 \times 10^{-4}$ (corresponding to $\alpha$ $=10$ and $\rho=0.1$ ). Figures $1(\mathrm{a})$ and 2-4 are histograms of $r_{k}$ as a function of $k$ at different times, and Fig. 5 records the time-dependent behavior of the average cluster size $\langle k\rangle$.

Figure 1(a) depicts an early stage of the kinetics. The

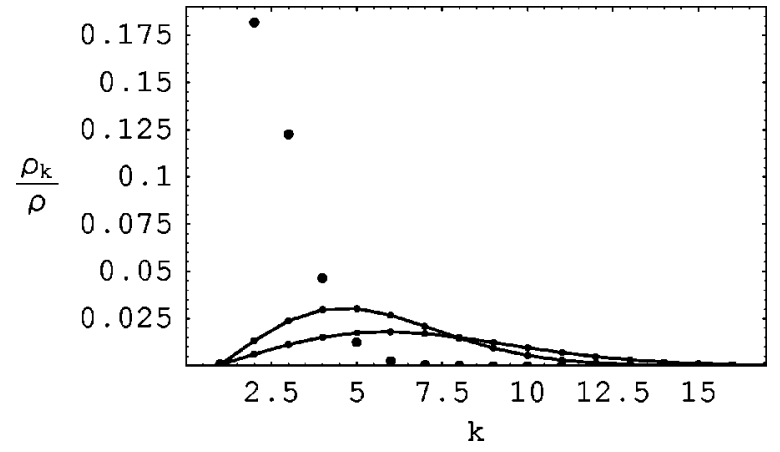

FIG. 2. Same as Fig. 1(a), for the times $\tau=20,10^{4}$, and $2 \times 10^{4}$. At the two later times, we have joined values of $\rho_{k} / \rho$ corresponding to neighboring $k$ 's by straight lines as a guide to the eye.

sequences of small dots at each $k$ record the values of $r_{k}$ at times between $\tau=0$ and $\tau=2$, in increments of $\Delta \tau=0.2$, and the larger dots joined by straight lines record the values of $r_{k}$ at $\tau=10$. The direction of increasing time is generally clear. As indicated in Fig. 1(b), the monomer concentration rapidly decreases to a small fraction of its initial value $r_{1}$ $=1$, so that the time orientation on the line $k=1$ is downward. Many small clusters of sizes $k(2 \leqslant k \leqslant 5)$ are simultaneously created, so the time orientation on the lines of these $k$ is generally upward. Notice that $\rho_{2}$ reaches a maximum and then decreases to a constant value, as can be seen in Fig. 1(c). By the end of the initial stage at time $\tau=10$, the creation of smaller clusters (with $2 \leqslant k \leqslant 5$ ) has slowed down greatly relative to the initial spurt for times $0<\tau<2$. Furthermore, the number of clusters with more than five monomers is negligible. At $\tau=10,\langle k\rangle \approx 2.69$, much smaller than the equilibrium value $\langle k\rangle \approx \sqrt{\rho e^{\alpha}}=\epsilon^{-1 / 2} \approx 46.9$. To determine the time scales appropriate for exploring the subsequent kinetics, it is highly instructive to plot the average cluster size $\langle k\rangle$ as a function of time, based on the numerical solution. Figure 5 is a $\log -\log$ plot of $\langle k\rangle / e$ as a function of $\tau$. It reveals an initial rapid growth of $\langle k\rangle$ to a "plateau value" close to $e$, roughly located in the interval $10<\tau$ $<100$. In the subsequent growth after the plateau, large clusters with $k \gg 1$ eventually appear. Figure 5 indicates that by time $\tau=5 \times 10^{4}, k$ clusters having $\langle k\rangle \approx 10$ are prevalent.

Figure 2 shows frames at times $\tau=20,10^{4}$, and $2 \times 10^{4}$,

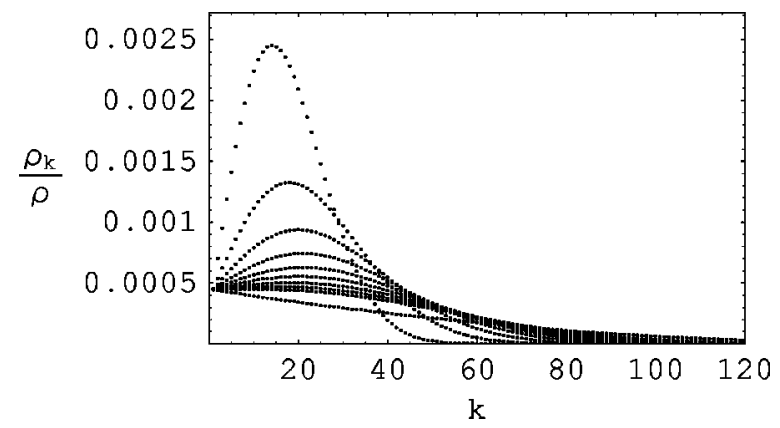

FIG. 3. Same as Fig. 1(a), starting at $\tau=2 \times 10^{5}$. Snapshots of the size distribution have been taken at time intervals of $\tau$ $=2 \times 10^{5}$, until a time $\tau=16 \times 10^{5}$. Then the last snapshot corresponds to $\tau=40 \times 10^{5}$. 


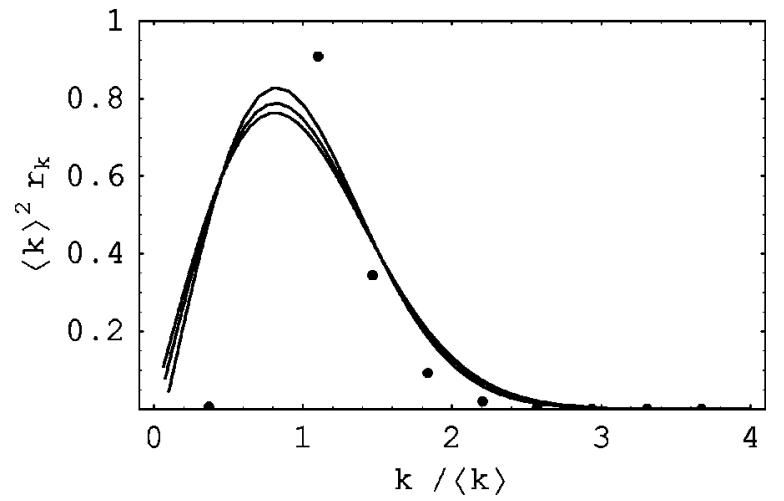

FIG. 4. Approximate self-similar behavior of the size distribution at times $\tau=50000,100000$, and 150000 (solid lines). Notice that $\langle k\rangle^{2} r_{k}$ is approximately the same function of $k /\langle k\rangle$ at different times. The dots correspond to $\tau=20$.

thereby continuing those in Fig. 1. The heavy dots correpond to $\tau=20$, which is well inside the plateau phase. The histograms at $\tau=10^{4}$ and $2 \times 10^{4}$ indicate the clear emergence of a continuum limit of the kinetics.

In the time interval $2 \times 10^{4}<\tau<5 \times 10^{5}$, the log-log plot of $\langle k\rangle / e$ as a function of $\tau$ in Fig. 5 is close to a straight line of slope $1 / 2$. This strongly supports the existence of a selfsimilar stage of the kinetics. The line graphs in Fig. 4 depict $\langle k\rangle^{2} r_{k}$ as a function of $x \equiv k /\langle k\rangle$ for the times $\tau=0.5 \times 10^{5}$, $10^{5}$, and $1.5 \times 10^{5}$. They are nearly superimposed on top of each other. The heavy dots correspond to the plateau time $\tau=20$, so the change in the distribution shape over the whole time span $20<\tau<1.5 \times 10^{5}$ is not very great.

The self-similar stage is not the final chapter of the kinetics story either. By $\tau=10^{6}$, the linear dependence of $\ln (\langle k\rangle / e)$ with $\ln \tau$ breaks down. In fact, at $\tau=10^{6},\langle k\rangle \approx 31.1$, which is comparable to the equilibrium value of 46.9 mentioned before. Evidently, there is a final stage of kinetics in which the size distribution asymptotes to its equilibrium form. Figure 3 is the final era of cluster aggregation, continued from Fig. 2, in which snapshots of the size distribution are taken at $\tau$ increments of $0.2 \times 10^{6}$, from $0.2 \times 10^{6}$ to $4 \times 10^{6}$. Conver-

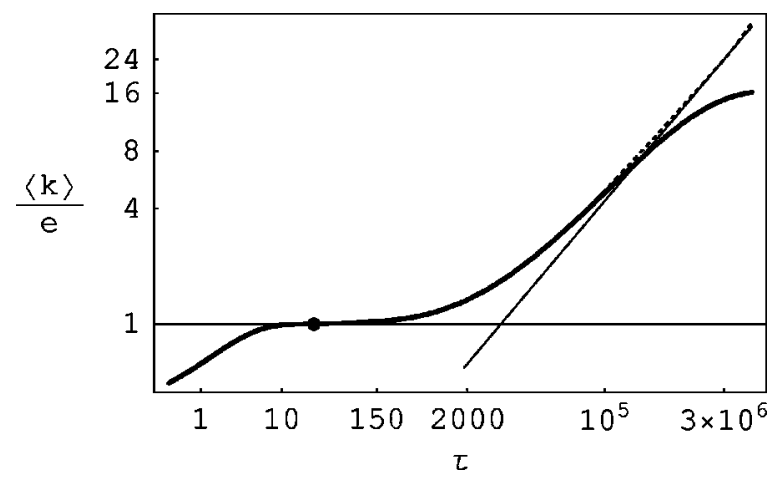

FIG. 5. Evolution of the average cluster size $\langle k\rangle / e$ as a function of the scaled time $\tau$ (thick solid line). The dotted line corresponds to the solution of the system (4.11) in Sec. IV below with an initial condition corresponding to the dot. The straight line of slope $1 / 2$ corresponds to the self-similar continuum size distribution given by Eq. (4.19). gence to an exponential distribution with $\langle k\rangle$ equal to the equilibrium value of 46.9 is clear.

\section{ASYMPTOTIC THEORY OF MICELLIZATION}

In this section, we shall interpret the numerical results shown in Sec. III by using singular perturbation methods; see Ref. [14] for a general description thereof.

\section{A. Initial transient}

Initially, $r_{1}(0)=1$, and there are no multiparticle aggregates. As we have seen in Sec. III, the numerical solution of the complete model shows that there is an initial transient stage during which dimers, trimers, etc. form at the expense of the monomers, and that $r_{k} \approx 0$ for sufficiently large $k$. Taking the $\epsilon \rightarrow 0$ limit of Eqs. (2.30) and (2.31) yields the following planar dynamical system:

$$
\begin{gathered}
\frac{d r_{1}}{d s}=-\left(r_{1}+r_{c}\right), \\
\frac{d r_{c}}{d s}=-r_{c}, \\
\frac{d s}{d \tau}=r_{1},
\end{gathered}
$$

in the adaptive time $s=\int_{0}^{\tau} \rho_{1} d \tau$. The general solution of the linear system (4.1) and (4.2) is

$$
r_{1}=(a-b s) e^{-s}, \quad r_{c}=b e^{-s},
$$

where $a$ and $b$ are arbitrary constants. Our initial condition yields $a=b=1$, so that

$$
r_{1}=(1-s) e^{-s}, \quad r_{c}=e^{-s},
$$

and from Eq. (4.3),

$$
\tau=\int_{0}^{s} \frac{e^{s}}{1-s} d s
$$

Clearly, $\tau \rightarrow \infty$ corresponds to $s \rightarrow 1-$. At $s=1$, Eq. (4.4) yields $r_{1}=0, r_{c}=e^{-1}$, which are the limiting values of the variables $r_{1}$ and $r_{c}$ at the end of the initial stage. Equation (2.27) with $\epsilon=0$ becomes $d\left(r_{k} e^{s}\right) / d s=r_{k-1} e^{s}$, which can be solved recursively to yield

$$
r_{k}=\left(\frac{s^{k-1}}{(k-1) !}-\frac{s^{k}}{k !}\right) e^{-s} .
$$

As $\tau \rightarrow \infty, r_{k} \rightarrow(k-1) e^{-1} / k$ !. Since $r_{6}(1)=0.00255$, after the initial transient stage there are insignificant numbers of aggregates with more than five monomers. In fact, the average aggregate cluster size is $\langle k\rangle=1 / r_{c}=e$; whereas at equilibrium, $\langle k\rangle \sim \sqrt{\rho e^{\alpha}} \gg 1$. We therefore conclude that there must be successive transients on time scales much larger than $t=O(\epsilon)$. 


\section{B. Intermediate transient}

Examination of the exact equation (2.27) shows that when $r_{1}$ decreases to size $O(\epsilon)$, but $r_{2}, r_{3}, \ldots$ are of order 1 , all terms in its right hand side are $O(\epsilon)$. This suggests rescaling $r_{1}=\epsilon R_{1}$, so that $\rho_{1}=e^{-\alpha} R_{1}$, and using the original time $t$ $=\epsilon \tau$. Equation (2.27) becomes

$$
\begin{gathered}
\frac{d r_{2}}{d t}=-\left(R_{1}-1\right)\left(r_{2}-\epsilon R_{1}\right)+r_{3}-2 r_{2}+\epsilon R_{1}, \\
\frac{d r_{k}}{d t}=-\left(R_{1}-1\right)\left(r_{k}-r_{k-1}\right)+r_{k+1}-2 r_{k}+r_{k-1}, \quad k \geqslant 2 .
\end{gathered}
$$

The global identities (2.30) and (2.31) become

$$
\begin{gathered}
\left(R_{1}-1\right) r_{c}-r_{2}+\epsilon\left(\frac{d R_{1}}{d t}+R_{1}^{2}+R_{1}\right)=0 \\
\frac{d r_{c}}{d t}+\left(R_{1}-1\right) r_{c}+\epsilon R_{1}=0
\end{gathered}
$$

where now $r_{c}=\epsilon R_{1}+\sum_{k=2}^{\infty} r_{k} \sim \sum_{k=2}^{\infty} r_{k}$, as $\epsilon \rightarrow 0$. In the limit $\epsilon \rightarrow 0, R_{1}-1=r_{2} / r_{c}$, and Eq. (4.8) becomes

$$
\frac{d r_{k}}{d t}=-\frac{r_{2}\left(r_{k}-r_{k-1}\right)}{r_{c}}+r_{k+1}-2 r_{k}+r_{k-1}, \quad k \geqslant 2 \text {. }
$$

This is a closed system of equations for $r_{2}, r_{3}, \ldots$, to be solved with the asymptotic values $r_{k}=(k-1) e^{-1 / k}$ ! as initial conditions. It can be shown that the reduced versions of Eq. (4.10) $\left[\dot{r}_{c}=-\left(R_{1}-1\right) r_{c}\right]$ and the conservation condition $\sum_{k=2}^{\infty} k r_{k}=1$, are upheld automatically by the solution of Eq. (4.11), so that they are redundant for this stage.

The numerical solution of the reduced system of equations (4.11) for $r_{k}, k \geqslant 2$ closely approximates that of the full system of kinetic equations at this stage. It can be seen that more and more $r_{k}$ become different from zero as $t$ increases, and that $r_{k}-r_{k-1}$ becomes small. This strongly suggests that $r_{k}$ can be approximated by a continuum limit for long times. To find the continuum limit, we set

$$
r_{k}(t) \sim \delta^{a} r(x, T), \quad x=\delta k, T=\delta^{b} t .
$$

Here $\delta \rightarrow 0$ fixes the scale of $k=O(1 / \delta)$, so that $x$ is fixed at some value of order $1 ; a$ and $b$ are positive exponents to be determined. To find $a$, we use the conservation condition $\sum_{k=2}^{\infty} k r_{k}=1$ :

$$
1=\delta^{a-2} \sum_{k=2}^{\infty}(k \delta) r(k \delta, T) \delta \sim \int_{0}^{\infty} x r(x, T) d x
$$

provided $a=2$. The limiting form of the particle conservation is thus

$$
\int_{0}^{\infty} x \operatorname{rr}(x, T) d x=1
$$

A similar calculation for the total number of clusters yields $r_{c} \sim \delta \int_{0}^{\infty} r(x, T) d x$, which suggests the definition

$$
r_{c} \sim \delta R_{c}, \quad R_{c} \equiv \int_{0}^{\infty} r(x, T) d x .
$$

We now substitute Eq. (4.12) in Eq. (4.11), and use Eq. (4.14) instead of $r_{c}$. The result is

$$
\begin{aligned}
\delta^{b} \frac{\partial r}{\partial T} \sim & -\frac{\delta^{2} r(2 \delta, T)[r(x, T)-r(x-\delta, T)]}{\delta R_{c}}+r(x+\delta, T) \\
& -2 r(x, T)+r(x-\delta, T) .
\end{aligned}
$$

The right hand side of this expression is of order $O\left(\delta^{2}\right)$, so that the following distinguished limit is obtained if we set $b=2$ and take $\delta \rightarrow 0$ :

$$
\frac{\partial r(x, T)}{\partial T}=-\frac{r(0, T)}{R_{c}(T)} \frac{\partial r(x, T)}{\partial x}+\frac{\partial^{2} r(x, T)}{\partial x^{2}} .
$$

For $k=2$, Eq. (4.11) and the scaling (4.12) with $a=b=2$ imply that $r(0, T)=0$. Therefore Eq. (4.15) becomes the simple diffusion equation

$$
\frac{\partial r}{\partial T}=\frac{\partial^{2} r}{\partial x^{2}}
$$

for $x>0, t>0$ to be solved with the boundary condition $r(0, T)=0$.

The numerical solution of the discrete equations (4.11) show that large aggregates do not emerge until $t \gg 1$. This suggests that the appropriate solution of Eq. (4.16) should be concentrated about $x=0$ as $T \rightarrow 0+$. That solution is proportional to the $x$ derivative of the diffusion kernel,

$$
r(x, T)=-\frac{\partial}{\partial x}\left(\frac{e^{-x^{2} / 4 T}}{\sqrt{\pi T}}\right)=\frac{x}{2 \sqrt{\pi} T^{3 / 2}} \exp \left(-\frac{x^{2}}{4 T}\right) .
$$

The numerical prefactor is chosen so that particle conservation, given by Eq. (4.13), holds. It follows from Eq. (4.14) that $R_{c}=(\pi T)^{-1 / 2}$. Hence the average aggregate size is

$$
\langle k\rangle=\frac{1}{\delta R_{c}}=\frac{\sqrt{\pi T}}{\delta} .
$$

In terms of the original variables $k, t$, and $r_{k}$, the previous expressions are

$$
r_{k}(t) \sim \frac{k}{2 \sqrt{\pi} t^{3 / 2}} \exp \left(-\frac{k^{2}}{4 t}\right),
$$

$$
\langle k\rangle \sim \sqrt{\pi t}
$$

as $t \rightarrow \infty$. These two equations yield

$$
\langle k\rangle^{2} r_{k} \sim \frac{\pi k}{2\langle k\rangle} \exp \left[-\frac{\pi}{4}\left(\frac{k}{\langle k\rangle}\right)^{2}\right],
$$


which resembles the behavior of the numerical solution of the full kinetic model as indicated in Fig. 4. Notice that the average cluster size $\langle k\rangle$ corresponding to the solution of Eqs. (4.11) (dotted line in Fig. 5) approaches the value (4.20) (straight line of slope 1/2 in Fig. 5).

\section{Equilibrium transient}

The large time limit of Eq. (4.19) does not match the equilibrium size distribution, which is $r_{k} \sim \epsilon e^{-k \sqrt{\epsilon}}$ in the same scaled units; see Sec. II. Thus the limit given by Eq. (4.19) is expected to break down when it predicts an average $\langle k\rangle$ of the order of the equilibrium length $1 / \sqrt{\epsilon}$. According to Eq. (4.20), this occurs at a time $\sqrt{t}=O\left(\epsilon^{-1 / 2}\right)$, i.e., $t$ $=O\left(\epsilon^{-1}\right)$. In this third and final transient towards equilibrium, we set

$$
r_{k}(t)=\epsilon r(x, t), \quad x=\sqrt{\epsilon} k, \quad T=\epsilon t .
$$

This is the same scaling as in Eq. (4.12) with $a=b=2$ and $\delta=\sqrt{\epsilon}$, and therefore we use here the same notation for the variables. With this scaling, the scaled particle conservation is

$$
1=\sum_{k=1}^{\infty} k r_{k}=\epsilon^{1 / 2} \sum_{k=1}^{\infty} \epsilon^{1 / 2} k r(x, T)
$$

and the limit $\epsilon \rightarrow 0$ yields

$$
\int_{0}^{\infty} x r(x, T) d x=1 .
$$

Similarly,

$$
r_{c} \sim \epsilon^{1 / 2} \int_{0}^{\infty} r(x, T) d x \equiv \epsilon^{1 / 2} R_{c} .
$$

The scaled version of the global identity (2.32) is

$$
R_{c}\left(R_{1}-1\right)+\epsilon^{1 / 2} R_{1}+\epsilon \frac{d R_{c}}{d T}=0 .
$$

Here $r_{1}=\epsilon R_{1}=\epsilon r\left(\epsilon^{1 / 2}, T\right)$. It follows from Eq. (4.25) that

$$
R_{1}-1=-\frac{\epsilon^{1 / 2}}{R_{c}}+O(\epsilon)
$$

The scaled kinetic equation (2.27) is

$$
\begin{aligned}
\epsilon^{3} \frac{\partial r}{\partial T}= & -\epsilon^{2}\left(R_{1}-1\right)\left[r(x, T)-r\left(x-\epsilon^{1 / 2}, T\right)\right] \\
& +\epsilon^{2}\left[r\left(x+\epsilon^{1 / 2}, T\right)-2 r(x, T)+r\left(x-\epsilon^{1 / 2}, T\right)\right] .
\end{aligned}
$$

We now substitute Eq. (4.26) in this expression, divide it by $\epsilon^{3}$, and take the limit $\epsilon \rightarrow 0$. The result is

$$
\frac{\partial r}{\partial T}=\frac{1}{R_{c}(T)} \frac{\partial r}{\partial x}+\frac{\partial^{2} r}{\partial x^{2}} .
$$

In these units, the average aggregate length is $\langle x\rangle=1 / R_{c}$, and Eq. (4.27) can be rewritten as

$$
\frac{\partial r}{\partial T}=\langle x\rangle \frac{\partial r}{\partial x}+\frac{\partial^{2} r}{\partial x^{2}}
$$

to be solved with the boundary condition

$$
r(0, T)=1
$$

which follows from Eq. (4.26) with $\epsilon \rightarrow 0$. It can be straightforwardly checked that $(d / d T) \int_{0}^{\infty} x r(x, T) d x=0$, and therefore $\int_{0}^{\infty} x r(x, T) d x=1$, provided $r(x, 0)$ satisfies this particle conservation condition.

We now have to show two things: (1) As $T \rightarrow 0+$, the solution of Eqs. (4.28) and (4.29) is asymptotic [14] to the right hand side of Eq. (4.17), the self-similar limiting solution of the intermediate transient stage. (2) The solution of Eqs. (4.28) and (4.29) tends to the equilibrium size distribution as $T \rightarrow \infty$. Then the size distribution of the equilibration transient as $T \rightarrow 0+$ matches the long time limit of the previous intermediate stage, and tends towards equilibrium as $T \rightarrow \infty$. This completes the description of the dynamics of the aggregate size distribution.

\section{Matching with the intermediate transient stage}

We represent $r(x, T)$ as

$$
r(x, T)=\frac{1}{T} h(\zeta, T), \quad \zeta=\frac{x}{\sqrt{T}} .
$$

With prefactor $1 / T$, the particle conservation equation (2.6) and the total cluster density adopt the invariant forms

$$
\begin{gathered}
\int_{0}^{\infty} \zeta h(\zeta, T) d \zeta=1 \\
R_{c}(T)=\int_{0}^{\infty} r(x, T) d x=\frac{1}{\sqrt{T}} \int_{0}^{\infty} h(\zeta, T) d \zeta \equiv \frac{h_{c}(T)}{\sqrt{T}} .
\end{gathered}
$$

Then

$$
\langle x\rangle=\sqrt{\frac{T}{h_{c}(T)}} .
$$

Inserting this equation together with Eq. (4.30) in Eq. (4.28), we obtain

$$
\frac{\partial^{2} h}{\partial \zeta^{2}}+h+\frac{1}{2} \zeta \frac{\partial h}{\partial \zeta}=T\left(\frac{\partial h}{\partial T}+\frac{\zeta h}{h_{c}}\right),
$$

to be solved with the boundary condition indicated by Eqs. (4.29) and (4.30),

$$
h(0, T)=T
$$

Asymptotic similarity as $T \rightarrow 0$ means that $h(\zeta, T)$ in Eq. (4.30) has a limit $H(\zeta)$ as $T \rightarrow 0$. The limit equations obtained from Eqs. (4.31)-(4.35) are

$$
\frac{\partial^{2} H}{\partial \zeta^{2}}+H+\frac{1}{2} \zeta \frac{\partial H}{\partial \zeta}=0 \text { for } \zeta>0,
$$




$$
\begin{gathered}
H(0)=0 \\
\int_{0}^{\infty} \zeta H(\zeta) d \zeta=1 .
\end{gathered}
$$

The unique solution of these equations is $H(\zeta)$ $=\zeta e^{-\zeta^{2} / 4} /(2 \sqrt{\pi})$, which is the right hand side of Eq. (4.17).

\section{Trend towards equilibrium}

The stationary solution of Eq. (4.28) with the condition (4.29) is $r_{e}=e^{-x\langle x\rangle}$, and the particle conservation condition gives $\langle x\rangle^{2}=1$, so that $\langle x\rangle=1$. Then the stationary solution of Eq. (4.28) is $r_{e}=e^{-x}$, which is the sought equilibrium solution. To show that $r(x, T) \rightarrow r_{e}(x)$ as $T \rightarrow \infty$, we define the associated free energy

$$
\begin{gathered}
f[r]=\int_{0}^{\infty}\left[-r+r \ln \left(\frac{r}{r_{0}}\right)\right] d x-1, \\
r_{0}=e^{-x}
\end{gathered}
$$

and show that it is a Lyapunov functional for Eq. (4.28). Notice that $\int_{0}^{\infty} r \ln r_{0} d x=-\int_{0}^{\infty} x r d x=-1$, and therefore $f[r]$ is the usual free energy, $f[r]=\int_{0}^{\infty}(r \ln r-r) d x$.

First, the standard inequality $x \ln x \geqslant x-1$ for positive $x$ $=r / r_{0}$ yields $f \geqslant-\int_{0}^{\infty} e^{-x} d x-1=-2$, and therefore $f$ is bounded below. Notice that $f\left[r_{0}\right]=-2$ at equilibrium.

Second, time differentiation of Eq. (4.37) yields

$$
\frac{d f}{d T}=\int_{0}^{\infty} \frac{\partial r}{\partial T} \ln \left(\frac{r}{r_{0}}\right) d x
$$

If we now substitute Eq. (4.28), integrate by parts, and use $r(0, T)=r_{0}(0)=1$ and $\int_{0}^{\infty} r d x=1 /\langle x\rangle$, we obtain

$\frac{d f}{d T}=\langle x\rangle-\int_{0}^{\infty} \frac{1}{r}\left(\frac{\partial r}{\partial x}\right)^{2} d x=\langle x\rangle\left[1-\int_{0}^{\infty} r d x \int_{0}^{\infty} \frac{1}{r}\left(\frac{\partial r}{\partial x}\right)^{2} d x\right]$.

The right hand side of this equation is less or equal than zero because of the Cauchy-Schwarz inequality

$$
\begin{aligned}
1 & =r(0, T)^{2}=\left(\int_{0}^{\infty} \frac{\partial r}{\partial x} d x\right)^{2} \leqslant\left(\int_{0}^{\infty}\left|\frac{\partial r}{\partial x}\right| d x\right)^{2} \\
& \leqslant \int_{0}^{\infty} r d x \int_{0}^{\infty} \frac{1}{r}\left(\frac{\partial r}{\partial x}\right)^{2} d x .
\end{aligned}
$$

Therefore, we have proven that the free energy is a Lyapunov functional. We can rewrite Eq. (4.38) in an equivalent form by defining $\tilde{r}_{0}=\exp [-x\langle x\rangle]$, and using the identities

$$
\begin{gathered}
\langle x\rangle=\langle x\rangle^{2} \int_{0}^{\infty} r d x=\int_{0}^{\infty} r\left(\frac{\partial \ln \tilde{r}_{0}}{\partial x}\right)^{2} d x, \\
\langle x\rangle=-\langle x\rangle \int_{0}^{\infty} \frac{\partial r}{\partial x} d x=\int_{0}^{\infty} r \frac{\partial \ln r}{\partial x} \frac{\partial \ln \tilde{r}_{0}}{\partial x} d x,
\end{gathered}
$$

to obtain

$$
\frac{d f}{d T}=-\int_{0}^{\infty} r\left[\frac{\partial}{\partial x} \ln \left(\frac{r}{\tilde{r}_{0}}\right)\right]^{2}, \quad d x \leqslant 0
$$

This equation shows that $r \rightarrow \tilde{r}_{0}$ as $T \rightarrow \infty$. The particle conservation condition $\int_{0}^{\infty} x \tilde{r}_{0} d x=1$ yields $\langle x\rangle^{2}=1$, and therefore $\tilde{r}_{0}=e^{-x}$.

\section{Approximation of the size distribution function by matched asymptotic expansions}

An uniformly valid approximation to the size distribution function can be easily formed from (i) $r_{k}^{(1)}(\tau)$, given by Eqs. (4.5) and (4.6), (ii) $r_{k}^{(2)}(t)$, which solves the approximate system of equations (4.11), and $r_{c}=\sum_{k=2}^{\infty} r_{k}$ with the initial conditions $r_{k}(0)=(k-1) e^{-1 / k}$ !, and (iii) $r(x, T)$, which solves the nonlinear Fokker-Planck equation (4.28) with the condition (4.29), and it matches Eq. (4.17) as $T \rightarrow 0+$. The result is

$$
\begin{aligned}
r_{k}^{(\text {unif })}(\tau)= & r_{k}^{(1)}(\tau)+r_{k}^{(2)}(\epsilon \tau)+\epsilon r\left(\sqrt{\epsilon} k, \epsilon^{2} \tau\right)-\frac{k-1}{k ! e} \\
& -\frac{k}{2 \sqrt{\pi}(\epsilon \tau)^{3 / 2}} \exp \left(-\frac{k^{2}}{4 \epsilon \tau}\right)
\end{aligned}
$$

Figure 6 compares the distribution function given by Eq. (4.40) to the numerical solution of the complete model equations in times corresponding to the end of the intermediate stage and the beginning of the equilibration stage. At these times, $r_{k}^{(1)}=(k-1) /(k ! e)$. We observe a good agreement between approximate and numerical solutions, which improves as the time elapses and the equilibrium distribution is approached.

\section{CONCLUSIONS}

On the basis of a simple kinetic model and starting from the initial state of pure monomers, we have shown that the process of micellization of rodlike aggregates at high CMC occurs in three separated stages or eras. In the first era, many clusters of small size are produced while the number of monomers decreases sharply. During the second era, aggregates are increasing steadily in size and their distribution approaches a self-similar solution of the diffusion equation. Before the continuum limit can be realized, the average size of the nuclei becomes comparable to its equilibrium value, and a simple mean-field Fokker-Planck equation describes the final era until the equilibrium distribution is reached. A continuum size distribution does not describe micellization until the third era has started; during the first two eras the effects of discreteness dominate the dynamics.

In order to validate our theory by an experiment, it would be important to measure the average cluster size as a function 

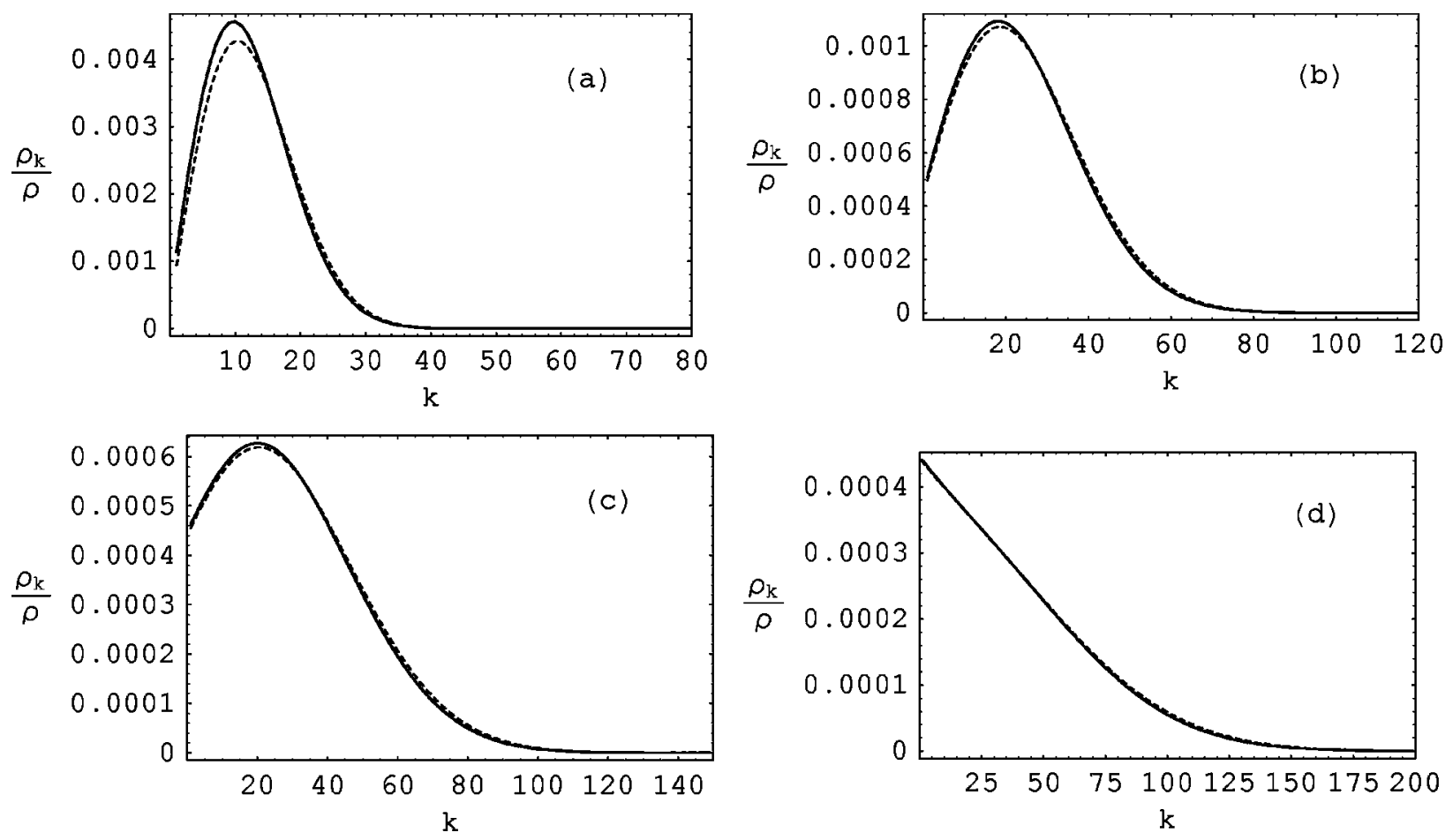

FIG. 6. Comparison of the approximation (4.40) (dashed line) to the numerical solution of the full kinetic model (solid line) for four different times $\tau$ : (a) 100000 , (b) 500000, (c) 1000000 , and (d) 3000000 . Notice that the agreement improves as the equilibrium distribution is approached.

of time, as in Fig. 5; the multiscale behavior is more clearly seen in this figure. To determine the time scale, we need a measure of the cluster diffusion coefficient $d$ that was set equal to 1 in Sec. II. A convenient relation could be Eq. (4.20), which in dimensional units is $\langle k\rangle \approx \sqrt{d \pi t}$. In case the self-similar size distribution is not reached during the intermediate phase, another way to determine $d$ is to study the equilibration era and compare the experimentally obtained size distribution with the numerical solution of the model. At equilibrium, $\langle k\rangle^{2} \approx \rho e^{\alpha}$, and this relation determines the dimensionless binding energy $\alpha$.

\section{ACKNOWLEDGMENTS}

We thank A. Carpio for helpful discussions on numerical solutions of the model. This work was carried out during a visit of J. Neu's to the Universidad Carlos III de Madrid, whose support we acknowledge. J. A. Cañizo was financially supported by the Spanish Ministry of Education. The present work was financed by the Spanish MCyT Grant Nos. BFM2002-04127-C02-01 and BFM2002-00831, by the Third Regional Research Program of the Autonomous Region of Madrid (Strategic Groups Action), and by the European Union under Grant No. RTN2-2001-00349.
[1] J.N. Israelachvili, Intermolecular and Surface Forces, 2nd ed. (Academic Press, New York, 1991).

[2] E.M. Lifshitz and L.P. Pitaevskii, Physical Kinetics (Pergamon Press, New York, 1981).

[3] U. Gasser, E.R. Weeks, A. Schofield, P.N. Pursey, and D.A. Weitz, Science 292, 258 (2001).

[4] I.M. Lifshitz and V.V. Slyozov, J. Phys. Chem. Solids 19, 35 (1961).

[5] S.Q. Xiao and P. Haasen, Acta Metall. Mater. 39, 651 (1991).

[6] S.P. Marsh and M.E. Glicksman, Acta Mater. 44, 3761 (1996).

[7] K.F. Kelton, in Solid State Physics, edited by H. Ehrenreich and D. Turnbull (Academic Press, New York, 1991), Vol. 45, p. 75.
[8] J.J.L. Velázquez, J. Stat. Phys. 92, 195 (1998).

[9] O. Penrose and A. Buhagiar, J. Stat. Phys. 30, 219 (1983); O. Penrose, J.L. Lebowitz, J. Marro, M. Kalos, and J. Tobochnik, ibid. 34, 399 (1984).

[10] V. Ganesan and H. Brenner, Phys. Rev. E 59, 2126 (1999).

[11] E.A.G. Aniansson and S.N. Wall, J. Phys. Chem. 78, 1024 (1974).

[12] R.J. Hunter, Foundations of Colloid Science (Oxford University Press, New York, 1987).

[13] S. Schmölzer, D. Gräbner, M. Gradzielski, and T. Narayanan, Phys. Rev. Lett. 88, 258301 (2002).

[14] J. Kevorkian and J. Cole, Multiple Scale and Singular Perturbation Methods (Springer, New York, 1996). 